\title{
Antiproliferative ability of a combination regimen of crocodile egg extract, wild radix ginseng and natural Ganoderma lucidum on acute myelogenous leukemia
}

\author{
CHUNG HIN CHUI ${ }^{1,2}$, RAYMOND SIU MING WONG ${ }^{2}$, GREGORY YIN MING CHENG ${ }^{2}$, FUNG YI LAU $^{2}$, \\ STANTON HON LUNG KOK ${ }^{1}$, CHOR HING CHENG ${ }^{1}$, FILLY CHEUNG ${ }^{1}$, WING KA TANG ${ }^{1}$, \\ IVY TUANG NGO TEO ${ }^{1}$, ALBERT SUN CHI CHAN ${ }^{1}$ and JOHNNY CHEUK ON TANG ${ }^{1}$
}

\begin{abstract}
${ }^{1}$ Cancer Drug Research and Development Laboratory, Central Laboratory of the Institute of Molecular Technology for Drug Discovery and Synthesis, State Key Laboratory of Chinese Medicine and Molecular Pharmacology, Department of Applied Biology and Chemical Technology, The Hong Kong Polytechnic University, Hung Hom, Kowloon;

${ }^{2}$ Division of Haematology, Department of Medicine and Therapeutics, Prince of Wales Hospital, The Chinese University of Hong Kong, Sha Tin, Hong Kong, P.R. China
\end{abstract}

Received March 30, 2006; Accepted June 29, 2006

\begin{abstract}
Chinese practitioners have employed the use of traditional Chinese medicine as an anti-cancer agent since the ancient period. Different combinations have been formulated for various purposes. Some have been claimed for postchemotherapy use but their direct actions on cancer cells may not be significantly reported. In the present study, we have tested the possible anti-leukemia potential of a combination regimen including crocodile egg extract, wild radix ginseng and natural Ganoderma lucidum (CGG extract) on acute myelogenous leukemia (AML) in vitro. A water soluble CGG extract was prepared and its antiproliferative activity was tested on the KG1a AML cell line and two freshly prepared bone marrow aspirate samples isolated from patients with de novo AML during presentation by a MTS/PMS assay. Furthermore, the possible activity of the CGG extract on the regeneration potential of $\mathrm{KG1a}$ cells was also investigated using a semi-solid methyl-cellulose colony formation assay. Lastly, the acute toxicity of CGG extract was further examined by a single high-dose oral feeding to rats. We found that the CGG extract could possess significant antiproliferative activity on AML cells. A strong colony formation inhibition was further demonstrated on KG1a cells. After feeding the rats with an excessive dose of CGG extract, we observed no development of acute toxicity. We concluded that
\end{abstract}

Correspondence to: Dr Johnny Cheuk On Tang, Department of Applied Biology and Chemical Technology, The Hong Kong Polytechnic University, Hung Hom, Hong Kong, P.R. China

E-mail: bccotang@inet.polyu.edu.hk

Key words: crocodile egg, Ganoderma lucidum, ginseng, leukemia, traditional Chinese medicine the CGG extract has growth inhibitory potential on KG1a cells and AML bone marrow samples in vitro. An in vivo toxicity test revealed that no acute toxicity was observed after feeding the rats a high dosage of the CGG extract. Further animal model tests are necessary to investigate the possible chronic toxicity of the CGG extract.

\section{Introduction}

Traditional Chinese medicine (TCM) is believed to have been used for at least five thousand years. Many commonly recognized TCM remedies for cancer treatment including ginseng have been extensively studied (1). Even though most of its active ingredients have been identified and purified, Chinese practitioners continue to use whole ginseng extract for adjunctive cancer therapeutic purposes. Ganoderma lucidum is another well-known TCM for cancer treatment. G. lucidum extract acts to induce growth inhibition plus apoptosis in breast cancer cell line MCF-7 and prostate cancer cell line PC-3 (2-3). The ratio of the apoptotic protein bax to the two anti-apoptotic proteins bcl-2 and bcl- ${ }_{\mathrm{xL}}$ was markedly increased after G. lucidum extract incubation. The apoptotic mechanism further involved cell cycle arrest together with change in cyclin level.

The combination of crocodile egg extract (33\%), wild radix ginseng (34\%) and natural G. lucidum (33\%) (CGG extract) has been suggested for individuals with poor immunity, deteriorating health, illness due to accumulated hard work, blood deficiency, physical enervation and fatigued patients especially after chemotherapy, radiotherapy and operation. However, the possible direct interaction of this combination towards acute myelogenous leukemia (AML) cells remains uncertain. Here we reported the experimental results of the CGG extract on the KG1a AML cell line plus primary cultured bone marrow cells isolated from de novo AML patients. Furthermore, the acute toxicity of the CGG extract was observed by a single-dose oral feeding to rats. Our results 
suggested that the CGG extract has an antiproliferative effect on AML cells and no acute toxicity was found after feeding rats with an excessive dose of the CGG extract.

\section{Materials and methods}

Preparation of the CGG extract for the in vitro antiproliferative activity test. The CGG extract sample was manufactured by Hong Kong Health and Long Biotechnology Ltd. and provided by Wah Sze Health Products Ltd. in capsule form. The powder was first dissolved in warmed distilled water and solubilization was further facilitated by sonication. Afterwards, the aqueous solution was filtered and warmed with absolute ethanol using an evaporator. Lastly, the remaining powder was weighed and adjusted to a final concentration with complete medium of $5000 \mu \mathrm{g} / \mathrm{ml}$. The clear solution was then filtered using a $0.22-\mu \mathrm{m}$ syringe filter. The final solution of the CGG extract was then stored at $-80^{\circ} \mathrm{C}$ until use.

Cell line and cell culture. The acute myelogenous leukemia (AML) cell line KG1a was obtained from American Type Culture Collection. It was maintained in RPMI-1640 medium (JRH Biosciences) supplemented with $10 \%$ of heatinactivated fetal bovine serum (Hyclone) together with antibiotics involving penicillin and streptomycin.

Human bone marrow cell collection and isolation. Bone marrow cells were collected from consenting de novo AML patients during presentation by bone marrow aspirate methods according to the regulations of the institute. A total of two adult subjects were recruited. Immediately after collection, mononuclear cells were enriched by Ficoll plaque (General Electricity, Amersham) gradient centrifugation. Cells were washed twice with phosphate-buffered saline and resuspended in complete medium. Viable cell percentage was then estimated by a trypan blue exclusion assay and counted using a hemacytometer.

[3-(4,5-Dimethylthiazol-2-yl)-5-(3-carboxymethoxyphenyl)2-(4-sulfophenyl)-2H-tetrazolium] (MTS) assay. Changes in the cellular viability of CGG-treated cells were monitored using the MTS activity assay as reported previously. Briefly, leukemia cells (KG1a and AML bone marrow aspirate samples) were seeded at a concentration of $1 \times 10^{5} / \mathrm{ml}$. After $6 \mathrm{~h}$, CGG was diluted in complete medium and they were added at the serial diluted concentrations. After $72 \mathrm{~h}$ of incubation, medium was removed and the MTS/PMS solution dissolved in PBS was added, and they were further incubated for exactly $30 \mathrm{~min}$. Afterwards, the optical absorbance was determined at $490 \mathrm{~nm}$ according to the user's manual with minor modifications (Promega).

Morphological monitoring of the CGG extract-treated colonies. KG1a leukemia cells were mixed with methylcellulose and incubated $\left(1 \times 10^{5} / \mathrm{ml}\right)$. The $500 \mu \mathrm{g} / \mathrm{ml} \mathrm{CGG}$ extract disc contained $10 \%$ of $5 \mathrm{mg} / \mathrm{ml} \mathrm{CGG} \mathrm{extract}$ dissolved in complete medium. The vehicle control consisted of $10 \%$ complete medium as placebo. Colony formation for both the CGG- and vehicle-incubated leukemia cells was recorded after 7 days.
Proteasome activity assay. KG1a cancer cells were treated with the CGG extract $(500 \mu \mathrm{g} / \mathrm{ml})$ for $24 \mathrm{~h}$. Total cellular protein was extracted and quantitated by the Bradford method. Then $30 \mu \mathrm{g}$ protein extract was mixed with the proteasome specific substrate (Suc-Leu-Leu-Val-Tyr-AMC, $20 \mu \mathrm{M}$ ) and reaction buffer and further incubated at $37^{\circ} \mathrm{C}$ for $1 \mathrm{~h}$. The resulting relative fluorescence units were then compared to the untreated control. Triplicate tests were performed for each experiment. A total of three experiments were conducted.

In vivo acute toxicity test. This part of the experiment was a consultancy study performed by the State Key Laboratory of Safety Evaluation on New Drugs in Guangzhou, P.R. China according to their regulations on animal testing. Briefly, a total of 40 rats were divided into two groups. Each group had 10 males and 10 females. For the CGG group, all of them were fed $8 \mathrm{~g} / \mathrm{kg}$ body weight of the CGG while the placebo group was fed only distilled water. Change in physical parameters including body weight were recorded for one week.

\section{Results}

CGG extract induced growth inhibition and reduced the colony formation potential of AML cells. After incubating the
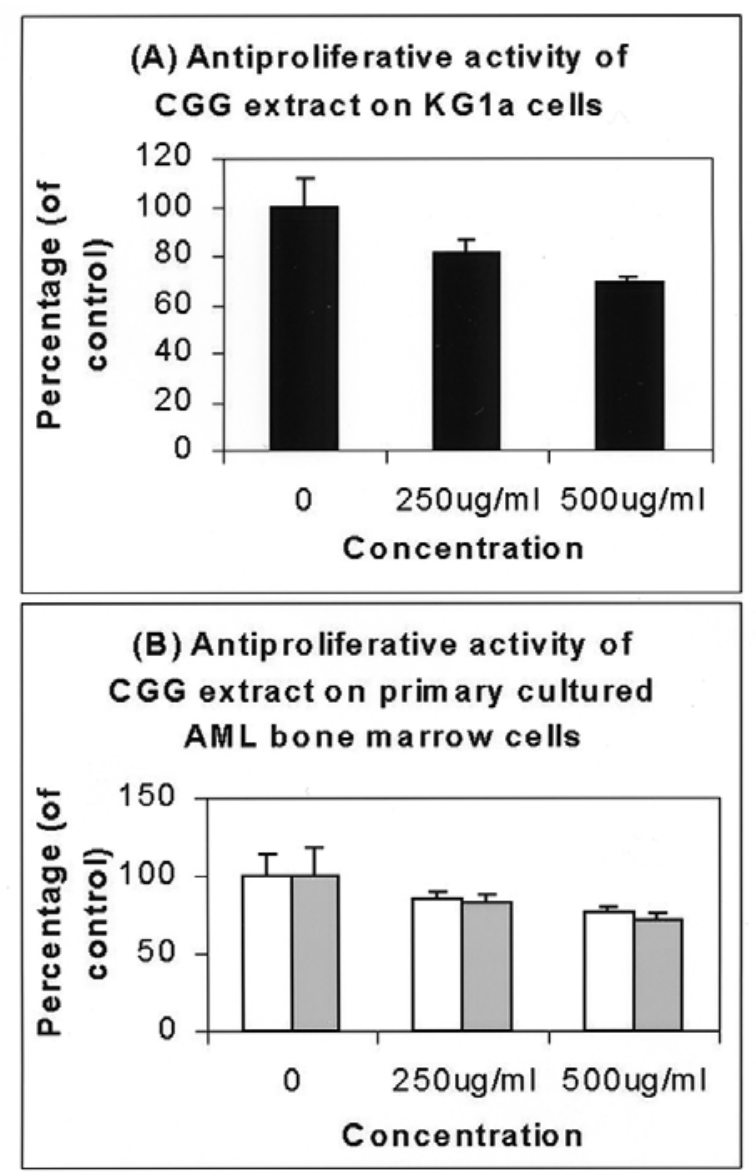

Figure 1. Antiproliferative effect of the CGG extract on (A) KG1a leukemia cell line and (B) two primary cultured AML bone marrow samples after $72 \mathrm{~h}$ of incubation and assay by MTS. Result are the mean \pm SD of one representative experiment. Triplicate tests were performed for each experiment. A total of three experiments were performed and similar results obtained. 

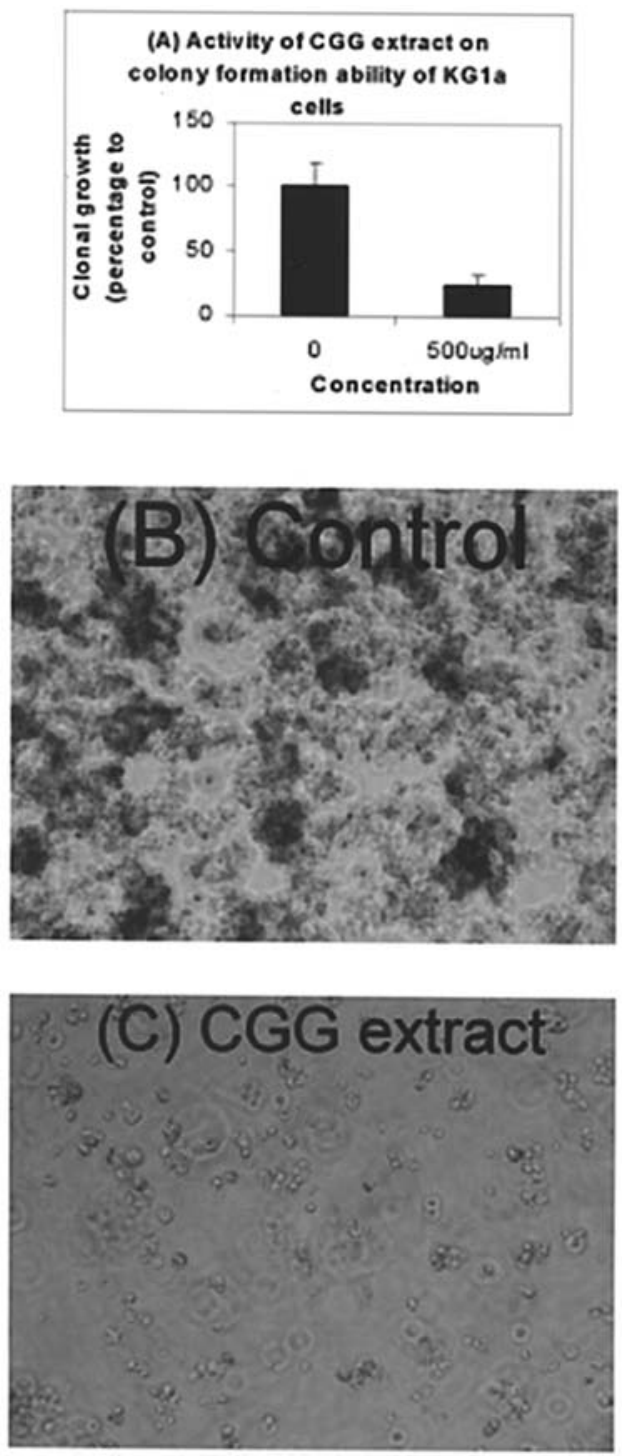

Figure 2. (A) Comparison of the colony formation ability of KG1a cells in the presence of the CGG extract. Results are the mean \pm SD of one representative experiment. Triplicate tests were performed for each experiment. A total of three experiments were performed and similar results obtained. (B) KG1a cells cultured with vehicle (10\% complete medium, v:v). (C) KG1a cells cultured with the CGG extract $(500 \mu \mathrm{g} / \mathrm{ml}$ in complete medium, $10 \% \mathrm{v}: \mathrm{v})$. A viable colony reduced the MTT to intracellular dark formazan.

KG1a cell line with the CGG extract for $72 \mathrm{~h}$, we observed that there was a significant antiproliferative effect exerted by the CGG extract on this leukemia cell line. The influence was dose-dependent (Fig. 1A). Similarly, the CGG extract was also active on the two bone marrow samples isolated from the AML patients (Fig. 1B).

To see whether continuous exposure of the CGG extract to KG1a cells would inhibit their regeneration potential, a colony formation assay was performed. As shown in Fig. 2, a strong inhibitory activity on the colony formation ability of the KG1a cells was observed.

KGla proteasome activity was reduced by the CGG extract. A significant reduction in relative fluorescence units from the CGG extract-treated cells ( $500 \mu \mathrm{g} / \mathrm{ml}$ for $24 \mathrm{~h})$ was observed. The relative mean percentage value dropped to $\sim 77 \%$ of the
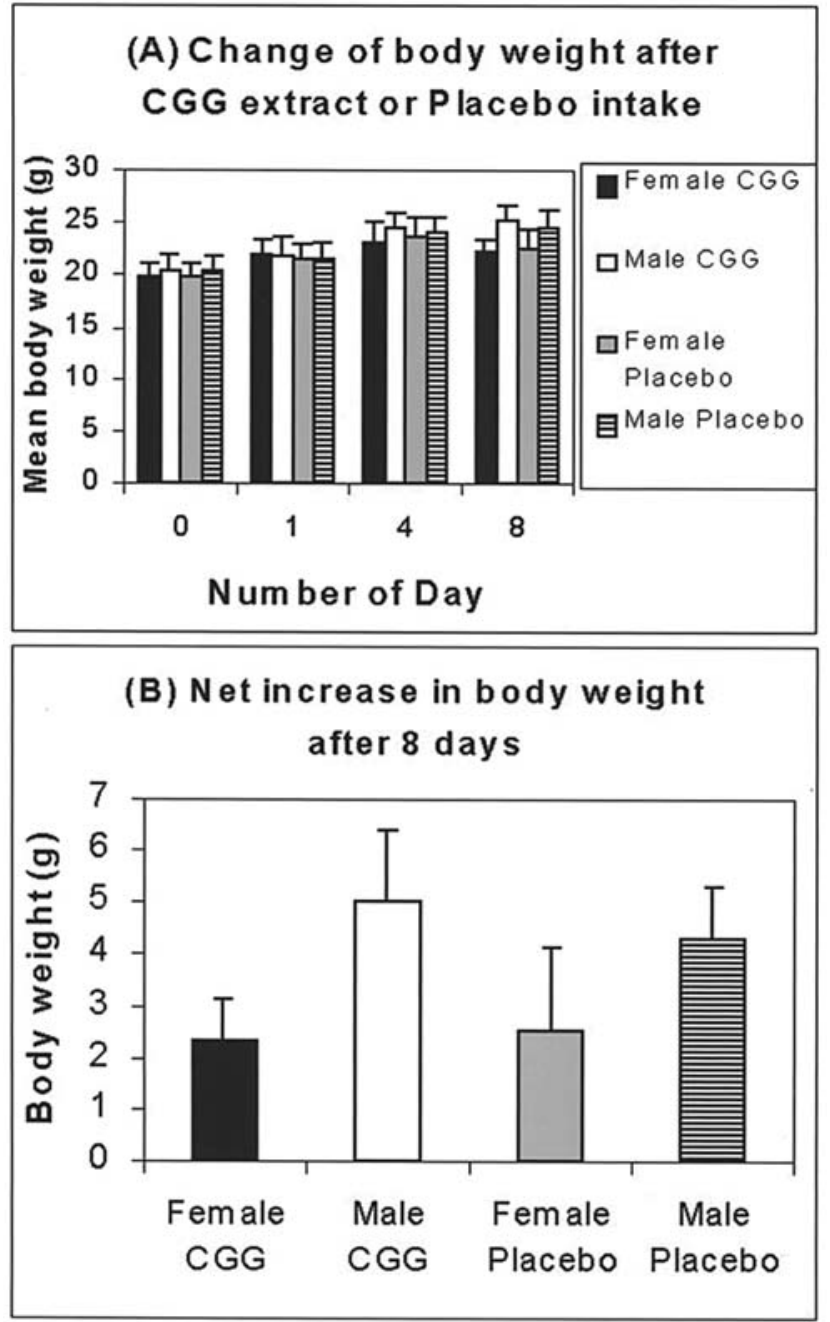

Figure 3. (A) Continuous monitoring of the changes in the animal body weight after a single high oral dose of the CGG extract. Each sex group had 10 rats. (B) Net change in body weight of the individual groups after 8 days. Each sex group contained 10 rats. Results are shown as mean \pm SD.

untreated control. Results were obtained from the mean of three independent experiments with each experiment performed in triplicate.

In vivo acute toxicity test. After feeding the rats with a high dose of the CGG extract ( $8 \mathrm{~g} / \mathrm{kg})$, no mortality was found. No abnormal behavior was detected. Investigation from the autopsy samples including the liver, the lung, and the reproductive and gastrointestinal systems revealed no pathological transformation. Lastly, there was a similar increment in the mean body weight of the rats in both the placebo and test groups (Fig. 3).

\section{Discussion}

Ginseng and G. lucidum have been used as a home remedy in TCM for a long time in South East Asia. Both are commonly used in alternative or supplemental cancer treatment. Recently, the use of G. lucidum on malignant cells of hematologic origin is especially attractive. Müller et al has performed an extensive investigation on the activity of the ethanol extract 
of G. lucidum on a panel of leukemia, lymphoma and myeloma cell lines in vitro. Their results strongly confirmed that $G$. lucidum may inhibit a distinct signal transduction pathway in different cancer cells (4). It is believed that the use of both ginseng and G. lucidum in cancer therapy is promising. The use of crocodile product as a medicine is also well recognized in China. Traditionally, the use of dried crocodile meat is an important therapeutic regimen in the treatment of asthma. The combination regimen including crocodile egg protein extract, ginseng and G. lucidum is commonly used to boost the immune response. Here, we asked whether such a combination could be active on AML cells including the KG1a cell line and bone marrow samples from patients.

We prepared a maximum aqueous extract concentration of $5000 \mu \mathrm{g} / \mathrm{ml}$. Using the MTS and colony formation assays, we confirmed that the CGG extract was active on both the KG1a cell line and two primary cultured AML samples. The antiproliferative activity was dose-dependent. Furthermore, a strong inhibition of regeneration potential on the KG1a cells was exerted by the CGG extract.

Proteasome is involved in mediating the protein degradation of many regulatory proteins concerned with normal physiological processes including programmed cell death and cell division (5). Therefore any naturally occurring or synthetic proteasome-specific inhibitors are believed to have potential use in cancer therapy. Recently, we demonstrated the proteasome inhibitory potential of Gleditsia sinensis fruit extract (6). Here we further demonstrated that the CGG extract could also contain potential natural proteasome inhibitor(s).

The major limitation to in vitro models is that cell cultures are only partly representative of in vivo conditions. As we know, the complexity of the metabolism of the human body and the interactions among constituents as well as many unknown factors cannot simply be predicted by cell lines and in vitro models (7). Here, our data demonstrated the fundamental step that the CGG extract was active on both the cell line and the primary culture of AML, and it is a potential proteasome inhibitor. This should provide us with a preliminary implication to further examine the possible antileukemia ability of the CGG extract.

Both acute and chronic toxicity is an important issue for the consumption of TCM remedies. In vivo animal experimental results suggested that the oral administration of the CGG extract at a dose of $8 \mathrm{~g} / \mathrm{kg}$ induced no development of acute toxicity on both sexes of rats. Assuming that an adult has a body weight of $50 \mathrm{~kg}$ with a total blood volume of 5 liters (8), the calculated concentration of the CGG extract, based on our in vitro data, would be $50 \mathrm{mg} / \mathrm{kg}$ in the human body. Thus theoretically our therapeutic concentration would still be far from the tested dose obtained from the animal experiment. Further SCID mice with an AML model might possibly explain whether oral administration of the CGG at the dosage predicted from in vitro data induces leukemia remission in vivo.

We have examined the anti-cancer potential of some TCM extracts including G. sinensis fruit (9), Radix Sophorae Tonkinensis, Scutellaria barbata (10) and Brucea javanica (11). All were found to be active in cancer of different origins including leukemia. Our results here further support the view that TCM could be an alternative choice for leukemia patients as a supplement to traditional chemotherapy (12).

\section{Acknowledgements}

This was a consultancy study requested by the Department of Medicine and Therapeutics, Prince of Wales Hospital, the Chinese University of Hong Kong (C.U.H.K), Department of Applied Biology and Chemical Technology, The Hong Kong Polytechnic University (H.K.P.U). This is an academic research study and should not be treated as 'advertisement' by any parties by any means. Drs F.Y. Lau and C.H. Chui were the honorary tutors kindly offered by Professors J.J.Y. Sung and G.Y.M. Cheng from the Department of Medicine and Therapeutics, Prince of Wales Hospital, The Chinese University of Hong Kong. Finally, Dr C.H. Chui would like to thank Professors A.S.C. Chan, K.Y. Wong, C.P. Lau, S.C.L. Lo and J.C.O. Tang who provided an opportunity for Dr C.H. Chui to be the 'Scientific Officer' in the Department of Applied Biology and Chemical Technology, H.K.P.U.

\section{References}

1. Keum YS, Park KK, Lee JM, Chun KS, Park JH, Lee SK, Kwon $\mathrm{H}$ and Surh YJ: Antioxidant and anti-tumor promoting activities of the methanol extract of heat-processed ginseng. Cancer Lett 150: 41-48, 2000.

2. Hu H, Ahn NS, Yang X, Lee YS and Kang KS: Ganoderma Incidum extracts induces cell cycle arrest and apoptosis in MCF-7 human breast cancer cell. Int J Cancer 102: 250-253, 2002.

3. Jiang J, Slivova V, Valachovicova T, Harvey K and Sliva D: Ganoderma lucidum inhibits proliferation and induces apoptosis in human prostate cancer cells PC-3. Int J Oncol 24: 1093-1099, 2004

4. Müller CI, Kumagai T, O'Kelly J, Seeram NP, Heber D and Koeffler HP: Ganoderma Incidum causes apoptosis in leukemia, lymphoma and multiple myeloma cells. Leuk Res (in press).

5. Lee DH and Goldberg AL: Proteasome inhibitors: Valuable new tools for cell biologists. Trends Cell Biol 8: 397-403, 1998.

6. Cheung F, Chui CH, Chan ASC, Lau FY, Cheng GYM, Wong RSM, Kok SHL, Teo ITN, Cheng CH and Tang JCO: Inhibition of proteasome activity in Gleditsia sinensis fruit extract mediated apoptosis on human carcinoma cells. Int J Mol Med 16: 925-929, 2005.

7. Farabegoli F: Green tea in human cancer. Minerva Biotec 17: 163-173, 2005.

8. Chui CH, Hau DKP, Lau FY, Cheng GYM, Wong RSM, Gambari R, Kok SHL, Lai KB, Tang JCO, Fong DWF and Chan ASC: Apoptotic potential of concentrated effective microorganism fermentation extract on human cancer cells. Int J Mol Med 17: 279-284, 2006

9. Chui CH, Lau FY, Chan ASC, Cheng GYM, Wong RSM, Lai KB, Kok SHL, Au Yeung TTL, Teo ITN, Yau MYC, Cheung F, Cheng $\mathrm{CH}$ and Tang JCO: Gleditsia sinensis fruit extractinduced apoptosis involves changes of reactive oxygen species level, mitochondrial membrane depolarization and caspase 3 activation. Int J Mol Med 15: 539-543, 2005.

10. Chui CH, Lau FY, Tang JCO, Kan KL, Cheng GYM, Wong RSM, Kok SHL, Lai PBS, Ho R, Gambari R and Chan ACS: Activities of fresh juice of Scutellaria barbata and warmed water extract of Radix Sophorae Tonkinensis on anti-proliferation and apoptosis of human cancer cell lines. Int J Mol Med 16: 337-341, 2005.

11. Lau FY, Chui CH, Gambari R, Kok SHL, Kan KL, Cheng GYM, Wong RSM, Teo ITN, Cheng CH, Wan TSK, Chan ASC and Tang JCO: Antiproliferative and apoptosis-inducing activity of Bruces javanica extract on human carcinoma cells. Int $\mathrm{J}$ Mol Med 16: 1157-1162, 2005.

12. Chui CH, Gambari R, Lau FY, Teo ITN, Ho KP, Cheng GYM, Ke B, Higa T, Kok SHL, Chan ASC and Tang JCO: Anti-cancer potential of traditional Chinese herbal medicines and microbial fermentation products. Minerva Biotec 17: 183-191, 2005. 